\title{
FINDING IRREDUCIBLE SUBMANIFOLDS OF 3-MANIFOLDS
}

\section{LUKE HARRIS AND PETER SCOTT}

If $M$ is a compact 3-manifold, then Kneser's theorem [2] tells us that $M$ is a connected sum of prime manifolds. For many problems, this reduces the study of compact 3-manifolds to that of compact prime manifolds. When $M$ is non-compact, one might hope that $M$ is a finite or an infinite connected sum of prime manifolds. However, in [4], Scott gives an example of a 3-manifold $M$ which is not a finite or an infinite connected sum of primes. Since his example is simply connected, we see that the problem is a geometric one rather than one of pathology of the fundamental group of the manifold.

If $M$ is a compact 3-manifold with indecomposable fundamental group, then $M$ is the connected sum of a prime manifold $N$ together with a simply connected manifold. $N$ is irreducible except when $N$ is a 2-sphere bundle over the circle, in which case $\pi_{1}(M)=\mathbb{Z}$, and so in either case there is an irreducible manifold with the same fundamental group as $M$. In [4], Scott proves the following somewhat weaker result when the 3-manifold is not compact.

THEOREM. If $G$ is the fundamental group of a 3-manifold, and $G$ is indecomposable and contains no elements of order 2 , then there exists a 3-manifold $N$ with fundamental group $G$ and $\pi_{2}(N)=0$.

Scott's proof of the theorem involves constructing an irreducible 3-manifold with fundamental group containing $G$, and taking a cover of this manifold to obtain $N$. He had to exclude elements of order two to ensure that $\pi_{2}(N)$ was zero. The more recent result, due to Meeks, Simon and Yau [3], that a cover of a $\mathbb{P}^{2}$-irreducible manifold is $\mathbb{P}^{2}$-irreducible, shows that $N$ is actually $\mathbb{P}^{2}$-irreducible.

The main result of this paper is the following theorem, which is essentially a generalisation of Scott's result.

THEOREM 1. Let $M$ be a 3-manifold with the property that we cannot write it as a non-trivial connected sum $M=M^{\prime} \# C$ with $C$ closed and irreducible. Then there is an irreducible submanifold $N$ of $M$ such that the natural map $\pi_{1}(N) \rightarrow \pi_{1}(M)$ is an isomorphism.

Remark. The result holds even if we can write $M=M^{\prime} \# C$ with $C$ a 2 -sphere bundle over the circle.

The hypotheses of this theorem are much less restrictive than are those of Scott in [4], except that the possibility of fake 3-balls embedded in $M$ is excluded. The proof

Received 9 July 1992.

1991 Mathematics Subject Classification 57M99.

Second author partially supported by NSF grant DMS 90-03974. 
is also much simpler than that in [4]. It seems to us that the theorem should still be true even if the Poincare conjecture is false and the hypothesis is modified to allow the 3-manifold to contain fake 3-balls, but we are unable to prove this.

If the Poincare conjecture is true, then the theorem is best possible, as the following result shows.

COROllary 2. If the Poincaré conjecture is true, then the following conditions on a 3-manifold $M$ are equivalent.

(i) $M$ has the fundamental group of an irreducible closed 3-manifold, or we cannot write $M$ as a non-trivial connected sum $M=M^{\prime} \# C$ with $C$ closed and irreducible.

(ii) There exists an irreducible 3-manifold $N$ with fundamental group isomorphic to $\pi_{1}(M)$.

We prove this corollary in Section 1. We also give an example to show that Theorem 1 cannot be easily strengthened. In Section 2, we prove Theorem 1 .

The work in this paper is part of the Liverpool $\mathrm{PhD}$ thesis of Luke Harris, completed under the supervision of Peter Scott in 1988. Since then, Harris obtained a job not in the academic world and has never had time to prepare this work for publication. Finally, Scott agreed to prepare this for publication, to avoid the complete disappearance of the work.

\section{An example and the corollary}

One might hope to improve Theorem 1 by showing that any 3-manifold $X$ is the connected sum of closed irreducible manifolds and a manifold $M$ such that we cannot write $M$ as a non-trivial connected sum $M=M^{\prime} \# C$ with $C$ closed and irreducible. However, we shall give an example showing that this is not the case.

If a 3-manifold $M$ is a connected sum, we see a family of 2-spheres $\Sigma$ embedded in $M$ corresponding to this. We find the summands by cutting along $\Sigma$ and attaching 3-balls to the resulting 2-sphere boundary pieces. Of course, the summands must be 3manifolds, and so the pieces into which $M$ is cut by $\Sigma$ must be 3 -manifolds. To ensure this, the family $\Sigma$ must be locally finite, which means that only finitely many 2 -spheres in $\Sigma$ intersect any compact subset of $M$.

The example of Scott mentioned earlier has an infinite family of 2-spheres $\Sigma$ embedded in it, but $\Sigma$ is not locally finite, and so we cannot cut along $\Sigma$ to obtain the manifold as a connected sum of primes. We use the same idea to construct our example.

Consider $\mathbb{R}^{3}$, and the $x$-axis $X$ embedded in $\mathbb{R}^{3}$. At each integer point of $X$, we construct a circle centred on this point, lying in the plane orthogonal to $X$. Let $L_{i}$ denote a regular neighbourhood of the circle for each integer point $i$ on $X . L_{i}$ is a solid torus.

We shall remove $L_{i}$ and glue in any compact 3-manifold with boundary a single torus, the resultant manifold being defined by the choice of manifold glued in and the choice of gluing homeomorphism between the two tori along which we glue. For example, if we remove $L_{0}$ and glue in a solid torus, then the resulting manifold will be $\mathbb{R}^{3}, \mathbb{R}^{3} \#$ (lens space) or $\mathbb{R}^{3} \# S^{2} \times S^{1}$, depending on how we glue in the solid torus. Similarly, we could glue in a knot space, or a more complicated manifold. 
Assume for the moment that for each $L_{i}$ we glue in a solid torus in such a way as to obtain a lens space summand, not $S^{3}$. Thus $M$ is the connected sum of $\mathbb{R}^{3}$ and infinitely many irreducible manifolds. There is an infinite family of 2 -spheres $\Sigma$ embedded in $M$ corresponding to the connected sum. Further, each 2 -sphere in $\Sigma$ must intersect $X$.

$X$ corresponds to the $x$-axis in $\mathbb{R}^{3}$. We shall remove from $M$ an open regular neighbourhood of the subset $(-\infty,-1) \cup(+1,+\infty)$ of $X$. Thus all that remains of $X$ is an arc $\lambda$ corresponding to $[-1,1]$. The new manifold $M^{\prime}$ has two boundary planes. Each of the 2 -spheres in $\Sigma$ can be isotoped in $M$ so as to intersect $\lambda$ and no other part of $X$. If we call such a modified family of 2 -spheres $\Sigma^{\prime}$, then $\Sigma^{\prime}$ is contained in $M^{\prime}$. We can cut along any finite subset of the $\Sigma^{\prime}$ to obtain a partial decomposition of $M^{\prime}$ as a connected sum containing $S^{2} \times S^{1}$ summands, but all of the 2-spheres in $\Sigma^{\prime}$ intersect $\lambda$, and so we cannot cut along all of them. Since any non-trivial 2 -sphere in any family of 2-spheres must intersect $\lambda$, we see that $M^{\prime}$ cannot be decomposed as a connected sum of closed manifolds together with a manifold which does not contain any submanifolds which are punctured non-trivial closed 3-manifolds.

Notice that the presence of boundary in $M^{\prime}$ is irrelevant, since we can identify the two boundary planes of $M^{\prime}$ together to remove all the boundary (and replace $\lambda$ with a loop) and still have the same problem. Also, the $M_{i}$ in the connected sum decomposition of $M$ can be any closed 3-manifolds, by suitable choice of the manifold to be glued in and the gluing map. For example, if the Poincare conjecture is false, then all the $M_{i}$ could be fake 3 -spheres.

Now we give the proof of Corollary 2 which shows that Theorem 1 is best possible if the Poincaré conjecture holds.

Corollary 2. If the Poincaré conjecture is true, then the following conditions on a 3-manifold $M$ are equivalent.

(i) $M$ has the fundamental group of an irreducible closed 3-manifold, or we cannot write $M$ as a non-trivial connected sum $M=M^{\prime} \# C$ with $C$ closed and irreducible.

(ii) There exists an irreducible 3-manifold $N$ with fundamental group isomorphic to $\pi_{1}(M)$.

Proof. By Theorem 1, it suffices to show that (ii) implies (i). To see this, suppose $M$ satisfies (ii) but not (i). Then $\pi_{1}(M)$ must contain the fundamental group $H$ of a closed irreducible 3-manifold. We know $\pi_{1}(M) \neq H$. This closed 3-manifold is irreducible, not the 3-sphere or a fake 3-sphere (assuming the Poincaré conjecture), and so $H$ is not trivial or infinite cyclic. Also, $\pi_{1}(N)$ contains $H$. But then a result of Epstein [1] shows that $N$ must split as a non-trivial connected sum, one summand being a closed 3-manifold with fundamental group $H$. Thus $N$ is not irreducible, since the other summand is not the 3 -sphere. This contradiction completes the proof of the corollary.

\section{Finding an irreducible submanifold of $M$}

In this section we prove Theorem 1 .

THEOREM 1. Let $M$ be a 3-manifold with the property that we cannot write it as a non-trivial connected sum $M=M^{\prime} \# C$ with $C$ closed and irreducible. Then there is an irreducible submanifold $N$ of $M$ such that the natural map $\pi_{1}(N) \rightarrow \pi_{1}(M)$ is an isomorphism. 
Before beginning the proof, we shall need the following definitions.

Definition. A submanifold $X$ of a 3-manifold $M$ is incompressible if $\partial X$ is incompressible in $M$.

REMARK. Then the natural map $\pi_{1}(X) \rightarrow \pi_{1}(M)$ is injective.

Definition. A 3-manifold $N$ is weakly irreducible if the manifold $\hat{N}$ obtained from $N$ by attaching a 3-ball to every boundary 2-sphere of $N$ is irreducible.

Definition. A chunk in a 3-manifold is a submanifold $X$ of $M$ which is connected, compact, incompressible and weakly irreducible.

Remark. With this definition, a (punctured) 2-sphere bundle over the circle is not a chunk.

Proof of Theorem 1. When $M$ is compact or has a compact core, the result is relatively uninteresting since we can apply the more useful result of Kneser's theorem. However, we give the proof since it sheds light on the case when $\pi_{1}(M)$ is infinitely generated.

So assume that $M$ has a compact core $X$ embedded in $M$. We see by compressing the boundary of $X$ that $X$ is the union of incompressible pieces and 1-handles, and if we further split along a maximal family of 2-spheres in each incompressible piece, we see that $X$ is a union of chunks joined by 1-handles and $S^{2} \times I$ 's. Some chunks may be punctured 3-spheres, and thus strictly speaking could be eliminated by a more careful choice of the 2-spheres used to separate the incompressible pieces into chunks, but this is not necessary to the construction.

The first step is to replace all the $S^{2} \times I$ 's with $D^{2} \times I$ 's. Clearly, this does not essentially change the map of the fundamental group of $X$ to $\pi_{1}(M)$. So now $X$ is a union of chunks and 1-handles. Let $C$ be one of the chunks. It may have a spherical boundary component $S$. Assume that it also has another boundary component $F$. We can find an embedded arc $\lambda$ in $C$ joining $S$ to $F$, and we may assume that the ends of $\lambda$ are attached where no 1-handles of $X$ are attached. Now we remove from $C$ a regular neighbourhood of $\lambda$. Since one end of $\lambda$ was attached to a 2-sphere boundary component of $C$, this does not essentially change the map of $\pi_{1}(C)$ to $\pi_{1}(M)$, or the map of $\pi_{1}(X)$ to $\pi_{1}(M)$. However, removing $\lambda$ effectively deletes $S$ as a boundary component of $C$, by attaching it, and all the 1-handles attached to $S$, to $F$. Thus $F$ is replaced by $F \# S$, which is homeomorphic to $F$. In fact, up to homeomorphism, what we have done is attach to $F$ all the 1-handles that were attached to $S$, and then cap off $S$ by gluing in a 3-ball, but in such a way that the new $X$ is embedded in $M$. We may repeat this procedure until each chunk of $X$ either has no spherical components, or the chunk has just one boundary component, which is a 2-sphere. In this last case $C$ must be a 3-ball, by our hypothesis on $M$ and the fact that $C$ is a chunk. Now an embedded 2-sphere $S$ lying in $X$ may be assumed to lie in a chunk by a cut and paste argument along the boundary of the chunks, and either the chunk is a 3-ball, in which case $S$ bounds a 3-ball, or else $S$ lies in a chunk with no boundary 2-spheres, in which case the chunk must be irreducible and so again $S$ bounds a 3-ball. This implies that $X$ is irreducible. 
So in the case when $M$ has a compact core, the result is quite easy. We shall follow a similar method when $M$ does not have a compact core by considering an exhausting sequence for $M$. In particular, we shall derive an exhausting sequence $M_{i}$ for $M$ so that each $M_{i}$ is a union of chunks, 1-handles and $S^{2} \times I$ 's. Further, each chunk of $M_{i}$ is contained in a chunk of $M_{i+1}$, and is the only chunk of $M_{i}$ contained in this chunk of $M_{i+1}$. We can apply the method used above in the compact core case to $M_{1}$, to find a compact $N_{1}$ contained in $M_{1}$ with the maps $\pi_{1}\left(N_{1}\right) \rightarrow \pi_{1}(M)$ and $\pi_{1}\left(M_{1}\right) \rightarrow \pi_{1}(M)$ being essentially the same. Now (since we have carefully chosen the exhausting sequence $M_{i}$ ) we shall be able to do the same construction on each $M_{i}$, giving an irreducible submanifold $N_{i}$, but in such a way that $N_{i}$ contains $N_{i-1}$. In particular, the arcs removed from the chunks to remove the 2-sphere boundary components of the chunks will not meet $N_{i-1}$. But then we shall have each $N_{i}$ irreducible, and thus $N$, which is the union of the $N_{i}$, will be irreducible. It then remains to show that the map $\pi_{1}(N) \rightarrow \pi_{1}(M)$ is an isomorphism.

It will be convenient to assume that if $M_{i}$ is an exhausting sequence for $M$, then $M_{i} \subset \stackrel{M}{i+1}_{i+1}$, for all $i$. We can do this by taking an exhausting sequence for the interior of $M$ rather than $M$ itself. Then the irreducible submanifold $N$ that we hope to find will be embedded in $\stackrel{M}{M}$.

So now let $M_{i}$ be an arbitrary exhausting sequence for $\stackrel{\circ}{M}$. The first step will be to arrange that each $M_{i}$ is a union of chunks, 1-handles and $S^{2} \times I$ 's, and that chunks of $M_{i}$ are contained in chunks of $M_{i+1}$. We first consider $M_{1}$.

The boundary of $M_{1}$ need not be incompressible in $M$. As usual, by following the method of Stallings in [5], we find a sequence of compressing discs for $\partial M_{1}$. If such a disc lies in $M_{1}$ then we cut along it, and if the disc lies in $M-M_{1}$ then we add a regular neighbourhood of it to $M_{1}$. Eventually we end up with a union of incompressible pieces $X$, and $X$ together with some 1-handles is a connected submanifold $M_{1}^{\prime}$ of $M$ such that $M_{1}^{\prime}$ contains $M_{1}$. We can make a new exhausting sequence for $M$ starting from $M_{1}^{\prime}$ by deleting any $M_{t}$ in the sequence which do not contain $M_{1}^{\prime}$. To see that $M_{1}^{\prime}$ is a union of chunks, 1-handles and $S^{2} \times I$ 's, simply cut $X$ along a maximal family of 2-spheres embedded in $X$.

Now consider a term $M_{i}$ in the exhausting sequence. Again we want to add discs and cut along discs to obtain $X_{i}$, a union of incompressible submanifolds of $M_{i}$, such that $X_{i}$ together with some 1-handles is $M_{i}^{\prime}$ which contains $M_{i}$. We also want each chunk of $M_{i-1}$ to be contained in a component of $X_{i}$. To arrange this, we need to make sure that the discs we cut along to obtain $X_{i}$ are disjoint from the chunks of $M_{i-1}$. But, of course, the chunks of $M_{i-1}$ are incompressible, so we can arrange by cut and paste that the intersection of any disc with the boundary of the chunks is empty, and thus that the disc does not intersect the chunks of $M_{i-1}$.

Finally, we want to cut the incompressible pieces of $X_{i}$ into chunks. We find a maximal family of non-parallel 2-spheres $\Sigma$ embedded in $X_{i}$, and perform cut and paste on the intersection of $\Sigma$ with the boundary of the chunks of $M_{i-1}$. Then we can assume that $\Sigma$ does not intersect the boundary of these chunks. Some 2-spheres of $\Sigma$ may be contained in chunks of $M_{i-1}$, but we delete these and add to $\Sigma$ all the spherical boundary components of the chunks of $M_{i-1}$. Since the chunks of $M_{i-1}$ are weakly irreducible, any 2-sphere lying in a chunk which is not parallel to a 2-sphere boundary component of that chunk is redundant in a decomposition of $X_{i}$ into chunks. Note that $\Sigma$ may contain redundant 2-spheres, but that we do not care about this, since it means only that some of the chunks into which $X_{i}$ is cut may be punctured 3-spheres. $\Sigma$ now contains all the 2-spheres used to separate $M_{i-1}$ into chunks, and so the chunks 
of $M_{i-1}$ are contained in the chunks into which $X_{i}$ is cut by $\Sigma$. Since, in fact, we want the chunks for $M_{i-1}$ to be contained in the interior of the chunks for $M_{i}$, if we cut $M_{i-1}$ along a 2 -sphere $S$ by removing a regular neighbourhood of the form $S \times(-1 /(i-1)$, $1 /(i-1))$, then we cut $M_{i}$ along a regular neighbourhood of $S$ of the form $S \times(-1 / i$, $1 / i)$.

We next want to ensure that different chunks of $M_{i}$ are contained in different chunks of $M_{i+1}$. Suppose that chunks $C_{i}, C_{i}^{\prime}, \ldots$ of $M_{i}$ are contained in a chunk $C_{i+1}$ of $M_{i+1}$. We shall simply add $C_{i+1}$ to $M_{\mathfrak{i}}$, thus replacing the chunks $C_{i}, C_{\mathfrak{t}}^{\prime}, \ldots$ with a single chunk $C_{i+1}$; similarly if chunks $C_{i}, C_{i}^{\prime}, \ldots$ are contained in any chunk $C_{j}$ of $M_{j}$ where $j>i$. This does not affect any of the other chunks of $M_{i}$, although of course it will change the 1-handles and $S^{2} \times I$ 's of $M_{i}$. Since every time we do this we reduce the number of chunks of $M_{i}$, we see that the process must stop eventually. We do this for $M_{1}$ and then for every $M_{i}$ in the exhausting sequence, obtaining a new exhausting sequence with the desired property that two or more chunks of $M_{i}$ are not contained in a single chunk of $M_{i+1}$.

We are now ready for the final refinement of the exhausting sequence $M_{i}$. Let $C_{t}$ be a chunk of $M_{i}$ for some $i$. Then, for all $j>i, C_{i}$ is contained in the interior of some chunk $C_{j}$ of $M_{j}$. For every $j>i$, we consider the components of $C_{j}-\dot{C}_{i}$. Let $R$ be such a component. Then $R \cap C_{i}=F$, a boundary component of $C_{i}$. Suppose $F$ is not a 2sphere, but that every other boundary component of $R$ is a 2 -sphere. This is exactly the situation we want to avoid. To deal with this, we simply replace $C_{i}$ by $C_{i} \cup R$ (and then take a new exhausting sequence containing the new $M_{i}$ ). Note that $R$ does not meet any of the other chunks of $M_{i}$, so this does not affect them, although it will change the 1-handles and $S^{2} \times I$ 's of $M_{i}$. The reason that this process must terminate is that every time we do it, we reduce the number of non-spherical boundary components of $C_{i}$, and without affecting the other chunks of $M_{i}$.

We can now show that the exhausting sequence has the properties needed to construct $N$, the irreducible submanifold of $M$ with the map $\pi_{1}(N) \rightarrow \pi_{1}(M)$ an isomorphism.

First consider $M_{1}$. We replace all the $S^{2} \times I$ 's of $M_{1}$ with $D^{2} \times I$ 's, which are 1handles, of course. Then we remove all the spherical boundary components of each chunk by removing an arc $\lambda$ for each such boundary component joining it to some other boundary component, exactly as we did previously when we were dealing with the case when $M$ had a compact core. So we obtain $N_{1} \subset M_{1}$ but $N_{1}$ is irreducible, for exactly the same reason as before. We have $\pi_{1}\left(N_{1}\right)=\pi_{1}\left(M_{1}\right)$, and further, the maps of $\pi_{1}\left(N_{1}\right)$ and $\pi_{1}\left(M_{1}\right)$ to $\pi_{1}(M)$ are essentially the same.

We want to do the same thing for all the $M_{i}$. Suppose we have $N_{i}$ irreducible with $N_{i} \subset M_{i} \subset \stackrel{\circ}{M}_{i+1}$. We want to find $N_{i+1}$ irreducible containing $N_{i}$. Clearly there is no problem with replacing the $S^{2} \times I$ of $M_{i}$ with 1-handles in such a way that the result still contains $N_{i}$.

Let $C_{i}$ be a chunk of $M_{i}$, and let $X_{i}$ be the associated chunk of $N_{i}$ created by removing arcs $\lambda$ running from spherical boundary components of $C_{t}$ to other boundary components of $C_{i}$. Then $C_{i}$ and $X_{i}$ are contained in a chunk $C_{i+1}$ of $M_{i+1}$. We want to remove the spherical boundary components of $C_{i+1}$ by removing arcs from $C_{i+1}$ that are disjoint from $N_{i}$.

Consider a component $R$ of $C_{i+1}-\dot{X}_{i}$. Let $F=R \cap X_{i}$. Then $F$ is not a 2-sphere. Also, not all of the other boundary components of $R$ are 2-spheres, since this would imply the existence of a component of $C_{i+1}-\dot{C}_{i}$ with this property, which we have disallowed by choice of our exhausting sequence. So consider a spherical boundary 
component $S$ of $R$. $R$ has at least one non-spherical boundary component $F^{\prime}$ which is not $F$, and we can find an arc $\lambda$ embedded in $R$ running from $S$ to $F^{\prime}$. Further, we can choose $\lambda$ to be disjoint from any 1-handles of $N_{i}$. Then as usual we remove a regular neighbourhood of $\lambda$ to delete $S$ from the boundary of $C_{i+1}$. We do this for all 2 -sphere boundary components of $R$, for all components of $C_{i+1}-\dot{X}_{i}$ and for all chunks of $M_{i+1}$ until we obtain $N_{i+1}$ which is irreducible.

Now we claim that $N=\bigcup_{i=1}^{\infty} N_{i}$ is the submanifold of $M$ that we are looking for. Clearly $N$ is irreducible, since all the $N_{i}$ are irreducible. $\pi_{1}(M)$ is the direct limit of the groups $\pi_{1}\left(M_{i}\right)$, with homomorphisms induced by the inclusions $M_{i} \rightarrow M_{j}, i \leqslant j$, and similarly $\pi_{1}(N)$ is the direct limit of the groups $\pi_{1}\left(N_{i}\right)$. We also have the maps $\phi_{i}: \pi_{1}\left(N_{i}\right) \rightarrow \pi_{1}\left(M_{i}\right)$ induced by inclusion, which are isomorphisms. These induce a unique map $\phi: \pi_{1}(N) \rightarrow \pi_{1}(M)$ which commutes with all the other maps and is an isomorphism. But then the natural map from $\pi_{1}(N)$ to $\pi_{1}(M)$ induced by the inclusion of $N$ in $M$ must be $\phi$, an isomorphism as required. This completes the proof of Theorem 1 .

\section{References}

1. D. B. A. Epstein, 'Projective planes in 3-manifolds', Proc. London Math. Soc. (3) 11 (1961) 469-484.

2. H. KNESER, 'Geschlossen Flächen in dreidimensionalen Mannigfaltigkeiten', Jahresber. Deutsch. Math.-Verein. 38 (1929) 258-260.

3. W. H. MeEkS III, L. SimON and S.-T. YAU, 'Embedded minimal surfaces, exotic spheres and manifolds with positive Ricci curvature', Ann. of Math. (2) 116 (1982) 621-659.

4. G. P. SCOTT, 'Fundamental groups of non-compact 3-manifolds', Proc. London Math. Soc. (3) 34 (1977) 303-326.

5. J. R. Stallings, 'On fibering certain 3-manifolds', Topology of 3-manifolds and related topics (PrenticeHall, Englewood Cliffs, NJ, 1962) 95-100.

Mathematics Department

University of Michigan

Ann Arbor, MI 48109

USA 